\title{
Pioglitazone may reduce cardiovascular events in high risk patients with prediabetes
}

Pioglitazone was incorrectly described as a PPAR-gamma inhibitor in this News story (BMJ 2016;352:11029, doi:10.1136/ bmj.i1029) when it is an agonist. The first sentence of the third paragraph should read that pioglitazone "improves insulin sensitivity by stimulating [not inhibiting] peroxisome proliferator activated receptor gamma."

Cite this as: BMJ 2016;352:11087

๑ BMJ Publishing Group Ltd 2016 